BMJ Open Diabetes

Research \& Care

\title{
Ceramides and phospholipids are downregulated with liraglutide treatment: results from the LiraFlame randomized controlled trial
}

\author{
Emilie H Zobel (D) , ${ }^{1}$ Asger Wretlind (D) , ${ }^{1}$ Rasmus S Ripa, ${ }^{2}$ \\ Viktor Rotbain Curovic (D) , ${ }^{1}$ Bernt J von Scholten, ${ }^{1,3}$ Tommi Suvitaival, ${ }^{1}$ \\ Tine W Hansen, ${ }^{1}$ Andreas Kjær, ${ }^{2}$ Cristina Legido-Quigley, ${ }^{1,4}$ Peter Rossing ${ }^{1}$
}

To cite: Zobel EH, Wretlind A, Ripa RS, et al. Ceramides and phospholipids are downregulated with liraglutide treatment: results from the LiraFlame randomized controlled trial. BMJ Open Diab Res Care 2021;9:e002395. doi:10.1136/ bmjdrc-2021-002395

\section{- Additional supplemental material is published online only. To view, please visit the journal online (http://dx.doi. org/10.1136/bmjdrc-2021- 002395).}

Presented at EASD 2020 and EDNSG 2021.

Received 17 May 2021 Accepted 25 August 2021

Check for updates

(c) Author(s) (or their employer(s)) 2021. Re-use permitted under CC BY-NC. No commercial re-use. See rights and permissions. Published by BMJ.

${ }^{1}$ Steno Diabetes Center Copenhagen, Gentofte, Denmark

${ }^{2}$ Department of Clinical Physiology, Nuclear Medicine \& PET and Cluster for Molecular Imaging, Rigshospitalet, Copenhagen, Denmark ${ }^{3}$ Novo Nordisk AS, Bagsvaerd, Denmark

${ }^{4}$ King's College, London, UK

Correspondence to

Dr Emilie H Zobel;

emilie.hein.zobel@regionh.dk

\section{ABSTRACT}

Introduction Treatment with glucagon-like peptide-1 receptor agonists (GLP-1 RAs) can reduce risk of cardiovascular disease (CVD) in persons living with type 2 diabetes, however the mechanisms explaining this cardiovascular benefit are still debated. We investigated changes in the plasma lipidome following treatment with the GLP-1 RA liraglutide.

Research design and methods In a double-blind placebo-controlled trial, we randomized 102 persons with type 2 diabetes to liraglutide or placebo for 26 weeks. Fasting blood plasma was collected at baseline and at end-of-treatment. The lipidome was measured using liquid-chromatography-coupled massspectrometry as a secondary end point in the study. Treatment response of each lipid was tested with lipid-specific linear mixed-effect models comparing liraglutide with placebo. Bonferroni $p<7.1 e-03$ was employed. The independence of the findings from clinical covariates was evaluated with adjustment for body mass index, $\mathrm{HbA}_{1 \mathrm{c}}$, fasting status, lipid-lowering treatment and change in lipid-lowering treatment during the trial.

Results In total, 260 lipids were identified covering 11 lipid families. We observed significant decreases following liraglutide treatment compared with placebo in 21 lipids $(p<7.1 e-03)$ from the following lipid families: ceramides, hexocyl-ceramides, phosphatidylcholines, phosphatidylethanolamines and triglycerides. We confirmed these findings in adjusted models $(p \leq 0.01)$. In the liraglutide-treated group, the individual lipids were reduced in the range of $14 \%-61 \%$ from baseline level, compared with $19 \%$ decrease to $27 \%$ increase from baseline level in the placebo group.

Conclusions Compared with placebo, liraglutide treatment led to a significant downregulation in ceramides, phospholipids and triglycerides, which all are linked to higher risk of CVD. These findings were independent of relevant clinical covariates. Our findings are hypothesis generating and shed light on the biological mechanisms underlying the cardiovascular benefits observed with GLP-1 RAs in outcome studies, and further strengthen the evidence base for recommending GLP-1 RAs to prevent CVD in type 2 diabetes.

Trial registration number NCT03449654.

\section{SIGNIFICANCE OF THIS STUDY \\ WHAT IS ALREADY KNOWN ABOUT THIS SUBJECT? \\ $\Rightarrow$ Glucagon-like peptide-1 receptor agonists (GLP-1 $\mathrm{RAs}$ ) can reduce the high risk of cardiovascular dis- ease in persons living with type 2 diabetes. \\ $\Rightarrow$ The mechanism behind this effect is largely unknown. \\ WHAT ARE THE NEW FINDINGS? \\ $\Rightarrow$ This is the first randomized placebo-controlled clin- ical trial to assess GLP-1 RAs' effect on the plasma lipidome using untargeted liquid-chromatography- coupled mass-spectrometry. \\ $\Rightarrow$ The present results suggested that treatment with the GLP-1 RA liraglutide led to a downregulation in ceramides, phospholipids and triglycerides. \\ $\Rightarrow$ Ceramides, phospholipids and triglycerides are lip- ids linked to risk of cardiovascular disease. \\ HOW MIGHT THESE RESULTS CHANGE THE FOCUS OF RESEARCH OR CLINICAL PRACTICE? \\ $\Rightarrow$ These results are hypothesis generating and may inspire research that aims to understand the car- diovascular mode-of-action of GLP-1 RAs to further investigate GLP-1 RAs' lipid-regulating effect.}

\section{INTRODUCTION}

Recent outcome studies have demonstrated that treatment with glucagon-like peptide-1 (GLP-1) receptor agonists can reduce the high risk of cardiovascular disease in persons living with type 2 diabetes. ${ }^{1-4}$ To date, there is no convincing evidence that points to a single mechanism as dominant for this risk reduction. $^{5}$

Favorable within-trial changes have been demonstrated in traditional cardiovascular risk factors including glycemia, body weight, systolic blood pressure, urinary albumin-tocreatinine ratio and low-density lipoprotein 
(LDL)-cholesterol. A recent analysis of The Liraglutide Effect and Action in Diabetes: Evaluation of Cardiovascular Outcome Results trial evaluated these cardiovascular risk factors as potential mediators of the effect of the GLP-1 receptor agonist liraglutide on major cardiovascular events. ${ }^{6}$ The analysis indicated potential mediation by $\mathrm{HbA}_{1 c}$ and to a lesser extent by urinary albumin-tocreatinine ratio. However, the authors concluded that $\mathrm{HbA}_{1 c}$ was unlikely to be a true mediator based on the totality of evidence including other cardiovascular outcome trials, where the choice of therapies used to reduce $\mathrm{HbA}_{1 \mathrm{c}}$, and not just $\mathrm{HbA}_{1 \mathrm{c}}$ reduction by itself, have important impact on cardiovascular outcomes. ${ }^{6}$

A mediation analysis can only evaluate candidate mediators that were measured during the trial. LDL-cholesterol was not found to be a mediator, but the lipid metabolism associated with type 2 diabetes and cardiovascular disease is complex, and other lipids play direct roles in development of atherosclerosis. With new lipidomic technologies using mass spectrometry, it is now achievable to study the whole range of lipids (lipidome) in a biological system. ${ }^{7}$ Lipidomics has been applied to discover new disease biomarkers and to unravel mechanisms and pathways underlying various diseases and therapies.

In a randomized, double-blind, placebo-controlled trial, we analyzed the lipidome before and after treatment with the human GLP-1 receptor agonists liraglutide as a secondary end point. Our aim was to explore downstream effects of liraglutide on lipid metabolism that could link to the cardiovascular benefit.

\section{RESEARCH DESIGN AND METHODS}

\section{Study design and participants}

The trial was conducted between October 26, 2017 and August 16, 2019 at the Steno Diabetes Center Copenhagen. We included men and women with type 2 diabetes in a randomized, double-blind, placebo-controlled, parallel-group trial. Participants were recruited from the outpatient clinic at the Steno Diabetes Center Copenhagen and through newspaper advertisements.

Inclusion criteria were as follows: age $>50$ years; $\mathrm{HbA}_{1 \mathrm{c}}$ $\geq 48 \mathrm{mmol} / \mathrm{mol}(6.5 \%)$; estimated glomerular filtration rate $\geq 30 \mathrm{~mL} / \mathrm{min} / 1.73 \mathrm{~m}^{2}$ (estimated by Chronic Kidney Disease Epidemiology Collaboration (CKDEPI) formula); stable glucose-lowering and cholesterollowering treatment (minimum 4 weeks). Key exclusion criteria were: type 1 diabetes; chronic or previous acute pancreatitis; other treatment (90 days prior to screening) which in the investigator's opinion could interfere with the effect of liraglutide (including oral glucocorticoids, calcineurin inhibitors, dipeptidyl peptidase 4 inhibitors, GLP-1 receptor agonists); cancer or disorders (except for conditions associated with type 2 diabetes history), which could interfere with the results of the trial; clinical signs of diabetic gastroparesis; previous bowel resection; impaired liver function; inflammatory bowel disease; weight $>150 \mathrm{~kg}$ (full list see online supplemental materials).

The primary end point of the trial was change in vascular inflammation assessed using ${ }^{18} \mathrm{~F}$-fluorodeoxyglucose PET $/ \mathrm{CT}^{8}{ }^{8}$ and here we report results from the secondary end point of change from baseline to week 26 in the lipidome for the liraglutide-treated group compared with placebo. The protocol for this analysis was developed before the last participant was enrolled in the study.

\section{Randomization and masking}

All participants were randomized in a 1:1 ratio to daily subcutaneous injections of liraglutide or matching placebo for 26 weeks. All investigators and participants were blinded to treatment allocation. Treatment pens and computer-generated allocation sequence were provided by Novo Nordisk (Bagsvaerd, Denmark) and two clinicians not otherwise involved in the trial handled the treatment allocation. EHZ enrolled participants and assigned participants to interventions.

\section{Procedures}

Starting dose was $0.6 \mathrm{mg} /$ day and was escalated to 1.2 $\mathrm{mg} /$ day after 1 week and to $1.8 \mathrm{mg} /$ day after 2 weeks. Maintenance-dose was $1.8 \mathrm{mg} /$ day. We allowed the dose escalation to be flexible in order to reach the maximum tolerated dose for each participant. Participants were instructed to be fasting for 4 hours prior to the study visits. Blood samples were collected at randomization and after 26 weeks treatment and were stored at $-80^{\circ} \mathrm{C}$ until analysis, which was 4 months after last patient last visit.

\section{Lipid quantification}

Plasma samples were prepared and analyzed at the Steno Diabetes Center Copenhagen. Lipids were extracted using a modified Folch extraction method ${ }^{9}$ and were analyzed by ultra-high-performance liquid chromatography coupled with quadrupole time-of-flight mass spectrometry. Samples were analyzed in a randomized order, with QC pooled plasma samples. Details can be found in the online supplemental materials.

The acquired data were preprocessed using MZmine 2 $(\mathrm{V} .2 .28)^{10}$ and postprocessed in R V.3.5.1 (R Core Team, Vienna, Austria) by normalization to internal standards, batch correction, truncation of outliers, imputation of missing values and $\log _{10}$ transformation. ${ }^{11}$

\section{Statistical analysis}

The lipidome analysis was prespecified and hypothesis generating. The sample size was a consequence of the sample size of the main study and no specific sample size estimation was done for this substudy.

Clinical data were assessed with SAS software (V.9.4; SAS Institute, Cary, North Carolina, USA). All data analysis and visualization related to the lipidome was done with R (V.3.5.1; R Core Team).

For the clinical data, continuous variables are given as means with $\mathrm{SD}$, non-normally distributed variables are 
Table 1 Characteristics of the participants at baseline

\begin{tabular}{|c|c|c|c|c|}
\hline & Total $(n=102)$ & Liraglutide $(n=51)$ & Placebo $(n=51)$ & $P$ value \\
\hline Sex (woman) & $16(15.7 \%)$ & $6(11.8 \%)$ & $10(19.6 \%)$ & 0.28 \\
\hline Age (years) & $66.4(8.2)$ & $65.9(8.6)$ & $66.9(7.8)$ & 0.56 \\
\hline Body mass index $\left(\mathrm{kg} / \mathrm{m}^{2}\right)$ & $29.9(4.6)$ & $30.5(5.3)$ & $29.3(3.8)$ & 0.16 \\
\hline \multicolumn{5}{|l|}{ Type 2 diabetes } \\
\hline Known duration in years & $10.9(5.7 ; 18.2)$ & $12.2(5.4 ; 18.2)$ & $10.2(5.7 ; 19.2)$ & 0.65 \\
\hline $\mathrm{HbA}_{1 \mathrm{c}}(\mathrm{mmol} / \mathrm{mol})$ & $58.4(10.1)$ & $58.7(9.6)$ & $58.0(10.6)$ & 0.73 \\
\hline \multicolumn{5}{|l|}{ Kidney function } \\
\hline Estimated glomerular filtration rate $\left(\mathrm{mL} / \mathrm{min} / 1.73 \mathrm{~m}^{2}\right)$ & $83.2(16.3)$ & $82.7(17.6)$ & $83.7(15.0)$ & 0.75 \\
\hline Urinary albumin-to-creatinine ratio (mg/g) & $6.0(3.5-14.5)$ & $6.0(3.5-15)$ & $6.0(3.5-14.5)$ & 0.65 \\
\hline \multicolumn{5}{|l|}{ Cardiovascular risk factors } \\
\hline Systolic blood pressure (mm Hg) & $135(17)$ & $133(15)$ & $137(20)$ & 0.25 \\
\hline Diastolic blood pressure $(\mathrm{mm} \mathrm{Hg})$ & $79(8)$ & $80(7)$ & $79(8)$ & 0.57 \\
\hline Total cholesterol (mmol/L) & $4.1(0.81)$ & $4.1(0.80)$ & $4.1(0.82)$ & 0.85 \\
\hline LDL-cholesterol (mmol/L) & $2.1(0.67)$ & $2.1(0.72)$ & $2.1(0.62)$ & 0.48 \\
\hline HDL-cholesterol (mmol/L) & $1.2(0.36)$ & $1.2(0.38)$ & $1.3(0.34)$ & 0.14 \\
\hline Triglycerides (mmol/L) & $1.8(1.0)$ & $2.1(1.2)$ & $1.6(0.78)$ & 0.01 \\
\hline Current smoker & $14(13.7 \%)$ & $10(19.6 \%)$ & $4(7.8 \%)$ & 0.08 \\
\hline \multicolumn{5}{|l|}{ History of cardiovascular event } \\
\hline Myocardial infarction & $13(12.8 \%)$ & $8(15.7 \%)$ & $5(9.8 \%)$ & 0.37 \\
\hline Stroke & $6(5.9 \%)$ & $3(5.9 \%)$ & $3(5.9 \%)$ & 1.00 \\
\hline Peripheral arterial thrombosis & $2(2.0 \%)$ & $2(3.9 \%)$ & $0(0.0 \%)$ & 0.50 \\
\hline \multicolumn{5}{|l|}{ History of cardiovascular symptoms } \\
\hline Claudication & $4(3.9 \%)$ & $3(5.9 \%)$ & $1(2.0 \%)$ & 0.62 \\
\hline Angina pectoris & $5(4.9 \%)$ & $4(7.8 \%)$ & $1(2.0 \%)$ & 0.36 \\
\hline Any cardiovascular disease & $23(22.6 \%)$ & $14(27.5 \%)$ & $9(17.7 \%)$ & 0.24 \\
\hline \multicolumn{5}{|l|}{ Glucose-lowering medication } \\
\hline Insulin use & $39(38.2 \%)$ & $20(39.2 \%)$ & $19(37.3 \%)$ & 0.84 \\
\hline SGLT2 inhibitors & $20(19.6 \%)$ & $8(15.7 \%)$ & $12(23.5 \%)$ & 0.32 \\
\hline \multicolumn{5}{|l|}{ Cardiovascular medication } \\
\hline Aspirin treatment & $37(36.3 \%)$ & $16(31.4 \%)$ & $21(41.2 \%)$ & 0.30 \\
\hline Statins and/or ezetimibe & $88(86.3 \%)$ & $46(90.2 \%)$ & $42(82.4 \%)$ & 0.25 \\
\hline Fibrates & $1(1.0 \%)$ & $1(2.0 \%)$ & $0(0.0 \%)$ & 1.00 \\
\hline
\end{tabular}

Adapted from Ripa et al. ${ }^{8}$ Data are $\mathrm{n}(\%)$, mean (SD) or median [IQR]. Differences in baseline characteristics between the liraglutide and the placebo group were tested using unpaired t-test and the $\chi 2$ test.

HDL, high-density lipoprotein; LDL, low-density lipoprotein; SGLT2, sodium glucose transporter 2.

summarized as medians with IQR and categorical variables are reported as percentages. Unpaired t-test and $\chi^{2}$ test was used to test differences in clinical characteristics between the two groups at baseline, and unpaired t-test was also used to compare change from baseline to endof-treatment in $\mathrm{HbA}_{1 \mathrm{c}}$, weight and clinical lipid measurements between the two groups.

To identify which lipid levels changed in response to treatment, linear mixed-effect models were built for each individual lipid species to explain observed standardized levels with time, treatment and their interaction as fixed effects and participant ID as random effect. A full list of lipids, estimated coefficients and $p$ values was reported.
Lipid class-level changes were assessed with the same approach of modeling on the average level in each lipid class. The independence of the findings from clinical covariates was evaluated with adjustment for body mass index (BMI), $\mathrm{HbA}_{1 c}$, fasting status, lipid-lowering treatment and change in lipid-lowering treatment during the trial by adding each of these covariates as additional independent variables to the linear mixed-effect model. Using similar approach, we evaluated the independence of the findings from the baseline measurement of total triglycerides.

Models were fitted using the limma ${ }^{12}$ and lme 4 packages. ${ }^{13}$ Nominal associations at $\mathrm{p}<0.05$ were reported 
for exploratory associations at the lipid class level also reported as family level. Bonferroni $\mathrm{p}<7.1 \mathrm{e}-03$ was employed for the single lipid linear mixed-effect models. Results were visualized as a lipidome-wide heatmap using the LipidomeR package. ${ }^{14}$

Selected lipid species $(n=21)$ with a response to the treatment in the linear mixed-effect models were investigated further: for each lipid species, relative change from baseline to end-of-treatment in each of the two arms was calculated. The result was visualized as a diverging bar graph with the ggplot2 package ${ }^{15}$ Baseline lipid levels were compared between participants with and without a history of cardiovascular disease. Standardized mean difference of the selected lipids was calculated using the 'effsize' package. ${ }^{16}$ Linear regression was used to investigate associations between the selected individual lipids and sex, age, BMI, $\mathrm{HbA}_{1 \mathrm{c}}$ and lipid-lowering treatment at baseline. Mediation analysis was performed with linear regression models, the significance and the tested mediation effect was calculated with the 'mediation' package in $\mathrm{R},{ }^{17}$ using bootstrapping to simulate 500 samples.

\section{Data availability statement}

The datasets analyzed during the current study are not publicly available due to the risk of patient re-identification. De-identified participant data or anonymized clinical study reports can be obtained from the first author on reasonable request. Necessary data protection agency and ethical committee approvals must be provided in compliance with relevant legislation.

\section{RESULTS}

\section{Participants}

The study included 102 participants, and all were eligible for lipidomic analysis. Five participants did not have blood samples available for lipidomic analysis at endof-treatment: one participant randomized to liraglutide dropped out of the study after experiencing gastrointestinal side effects, and one participant randomized to liraglutide discontinued the study treatment due to gastrointestinal side effects, remained in the study, but did not wish to have blood samples taken at the endof-treatment visit. Three participants randomized to placebo did not have blood samples taken at end-oftreatment: one dropped out of the study due to concurrent cancer disease, one discontinued study treatment after experiencing shoulder pain in relation to the first study visit, remained in the study, but did not wish to have blood samples taken at the end-of-treatment visit, and one did not show up for the visit where the end-oftreatment blood samples were taken due to work-related stress. These five participants were only included in the baseline analysis.

The population included $16(15.7 \%)$ women and the mean (SD) age was 66 (8.2) years, median (IQR) known diabetes duration was $10.9(5.7 ; 18.2)$ years, $\mathrm{HbA}_{1 \mathrm{c}} 58.4(10.1) \mathrm{mmol} / \mathrm{mol}$ and BMI 29.9 (4.6) kg/ $\mathrm{m}^{2}$. The baseline clinical characteristics of the 51 participants receiving liraglutide and the 51 receiving placebo were balanced (table 1), except for triglycerides (mean (SD): liraglutide $2.1(1.2)$ vs placebo $1.6(0.78) \mathrm{mmol} / \mathrm{L}$, $\mathrm{p}=0.01$ ). A history of cardiovascular disease (defined as history of acute myocardial infarction, percutaneous coronary intervention, coronary artery bypass graft, stroke, peripheral arterial thrombosis, claudication and/ or nitroglycerin requiring angina pectoris) was reported in $23(22.6 \%)$ participants at baseline (balanced between groups, $\mathrm{p}=0.24)$.

Mean changes $(95 \%$ CI) over the 26 weeks for the liraglutide-treated group compared with placebo in body weight were -3.7 ( -4.8 to -2.6$)$ vs -0.18 ( -0.76 to 0.40$) \mathrm{kg}$ $(\mathrm{p}<0.0001)$; and $\mathrm{HbA}_{1 \mathrm{c}}-5.1(-8.0$ to -2.0$)$ vs $-0.08(-1.9$ to 1.7$) \mathrm{mmol} / \mathrm{mol}(\mathrm{p}=0.006) .{ }^{8}$ Mean changes $(95 \% \mathrm{CI})$ for the liraglutide-treated group compared with placebo for clinical lipid measurements were as follows: LDLcholesterol $0.06(-0.17$ to 0.30$)$ vs $-0.08(-0.29$ to 0.13$)$ $\mathrm{mmol} / \mathrm{L} \quad(\mathrm{p}=0.37) ;^{8}$ HDL-cholesterol $0.06 \quad(0.004$ to $0.12)$ vs $0.05(-0.02$ to 0.11$) \mathrm{mmol} / \mathrm{L}(\mathrm{p}=0.72)$; total cholesterol 0.08 ( -0.18 to 0.35 ) vs -0.03 ( -0.26 to 0.21 ) $\mathrm{mmol} / \mathrm{L}(\mathrm{p}=0.54)$; triglycerides $-0.11(-0.44$ to 0.21$)$ vs $0.01(-0.19$ to 0.21$) \mathrm{mmol} / \mathrm{L}(\mathrm{p}=0.52)$.

\section{Lipids reduced by liraglutide treatment compared with placebo}

In total, 260 lipids were identified (figure 1, online supplemental table S1) covering 11 lipid families including ceramides, diglycerides, hexocyl-ceramides, lactosyl ceramides, lysophosphatidylcholines, lysophosphatidylethanolamines, phosphatidylcholines, phosphatidylethanolamines, phosphatidylinositoles, sphingomyelins and triglycerides. Of the 260 lipids, 21 were significantly decreased after liraglutide treatment compared with placebo (figure 1, online supplemental table S1). These 21 lipids were from the following lipid families: ceramides, hexocyl-ceramides, phosphatidylcholines, phosphatidylethanolamines and triglycerides. We observed a pattern with noticeably reduction in short ceramides and large, poly-unsaturated triglycerides (figure 1). The size of the reductions was ranging from $14 \%$ to $61 \%$ from baseline level, in the liraglutide-treated group, compared with $19 \%$ decrease to $27 \%$ increase from baseline level in the placebo group, as visualised in figure 2. We confirmed the reduction in these 21 individual lipids in models adjusted for BMI, $\mathrm{HbA}_{1 \mathrm{c}}$, fasting state (4 were not fasting for baseline measurements, and 3 were not fasting for end-of-treatment measurements), lipid-lowering treatment at baseline (yes or no) and change in lipid-lowering treatment during the study period $(\mathrm{n}=9)(\mathrm{p} \leq 0.01)$.

To provide a context for the 21 lipids that changed significantly in the liraglutide-treated group, we examined how they were related to selected baseline characteristics. At baseline, there were few correlations between these 21 individual lipids and sex, age, $\mathrm{BMI}, \mathrm{HbA}_{\mathrm{lc}}$ and lipid-lowering treatment (online supplemental table S2). The baseline levels of these 21 individual lipids were 

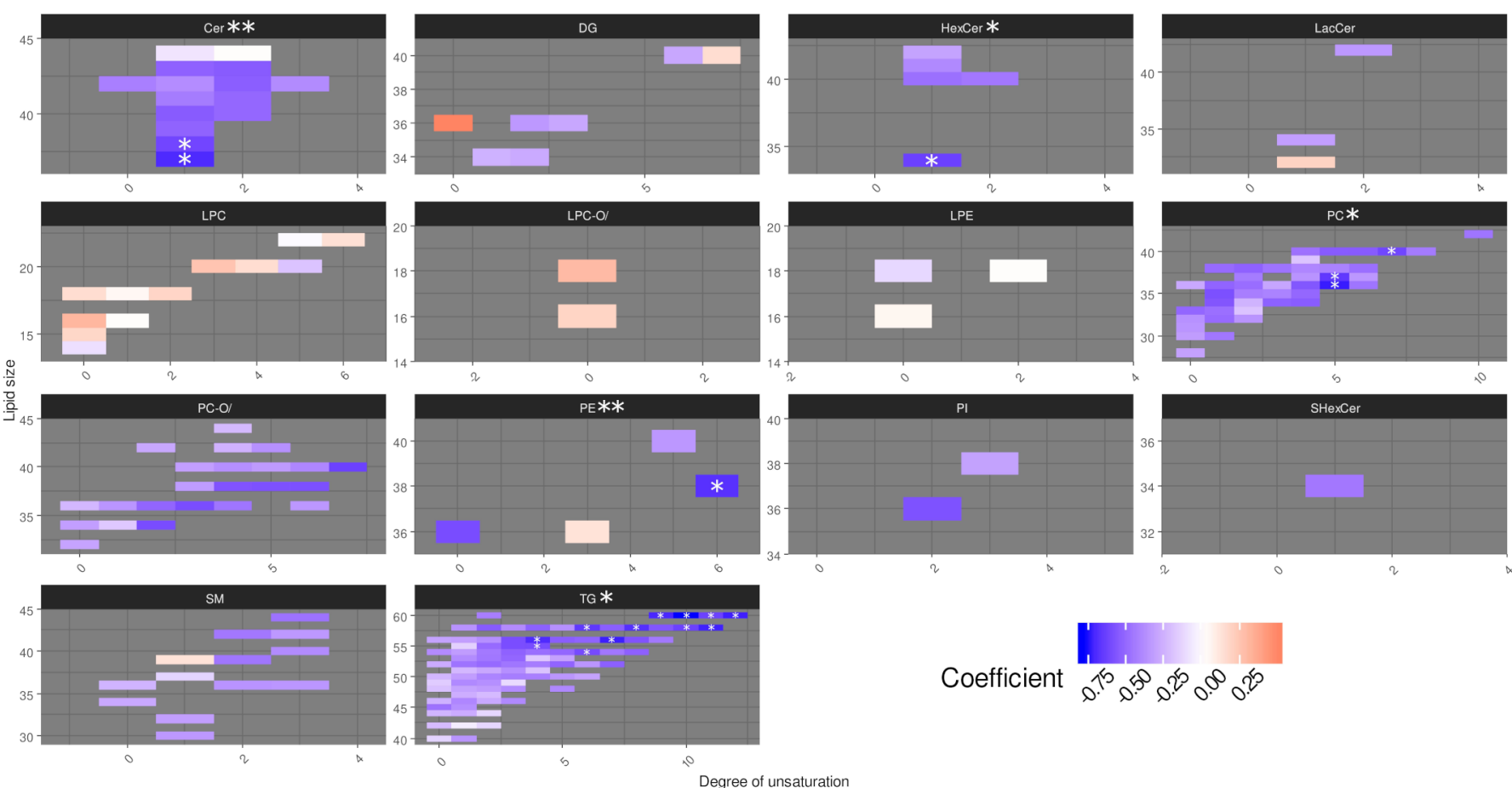

Figure 1 Lipidome-wide responses to liraglutide treatment compared with placebo. Lipid species grouped in panels according to lipid families (title of each panel). X-axis is the number of fatty-acid double bonds for the specific species (level of unsaturation) and Y-axis is the number of carbon atoms (total fatty acid chain length), and therefore each cell in the heat maps represents one lipid species (one individual lipid). Color indicates the coefficients from lipid-specific linear mixedeffect models. Blue colors represent lipids that are reduced in level by liraglutide treatment compared with placebo. Red colors represent lipids that are increased in level by liraglutide treatment compared with placebo. * $p<0.05$; ${ }^{* *} p<0.01$. Cer, ceramides; DG, diglycerides; HexCer, hexocyl-ceramides; LacCer, lactosyl-ceramides; LPC, lysophosphatidylcholines; LPE, lysophosphatidylethanolamines; PC, phosphatidylcholines; PE, phosphatidylethanolamines; PI, phosphatidylinositoles; SM, sphingomyelins; TG, triglycerides.

generally higher in participants with versus without a history of cardiovascular disease (figure 3).

We also analysed changes on a lipid family level for the liraglutide-treated group compared with placebo, and observed significant decreases in 5 of the 11 lipid families namely ceramides (26weeks average reduction liraglutide vs placebo: $-27 \%$ vs $8 \%, \mathrm{p}=0.01)$, hexocyl-ceramides $(-28 \%$ vs $-10 \%, \mathrm{p}=0.02)$, phosphatidylcholines $(-5 \%$ vs $2 \%, \mathrm{p}=0.04)$, phosphatidylethanolamines $(-14 \%$ vs $7 \%$, $\mathrm{p}=0.004)$ and triglycerides $(-27 \%$ vs $14 \%, \mathrm{p}=0.03)$.

The primary end point of the LiraFlame trial, change in vascular inflammation assessed using ${ }^{18} \mathrm{~F}$-fluorodeoxyglucose PET/CT, was unchanged. ${ }^{8}$

\section{Sensitivity analysis}

In a sensitivity analysis, we adjusted for the clinical measurement of total triglycerides at baseline, since this level was not balanced between the two groups, despite randomization. The change in the 21 lipid levels in the liraglutide-treated group remained significant compared with placebo after this adjustment, the highest adjusted $\mathrm{p}$ value was $0.009(\mathrm{PE}(\mathrm{O}-34: 2) / \mathrm{PE}(\mathrm{P}-34: 1))$.

Moreover, we performed a sensitivity analysis, where zero change from baseline to end-of-treatment was assumed for the five participants with lipidomics data missing at end-of-treatment. Overall results were similar, but six single lipids (TG(58:10); TG(60:11); Cer(d38:1); TG(54:6); TG(55:4) and PE(O-34:2)/PE(P-34:1)) were no longer significantly reduced by liraglutide treatment compared with placebo after multiple adjustment (online supplemental table S3).

\section{Mediation analysis}

In a mediation analysis including the top 10 most associated lipids, we tested if the observed effect of liraglutide on these lipids were mediated through change in BMI or $\mathrm{HbA}_{1 \mathrm{c}}$. None of the $\mathrm{p}$ values for mediation effect were significant ( $\mathrm{p} \geq 0.06$; online supplemental table $\mathrm{S} 4)$.

\section{DISCUSSION}

In this randomized clinical trial, we demonstrate that individual ceramide and phospholipid lipid species were reduced in the liraglutide-treated group compared with placebo. In addition, we confirmed a reduction in individual triglyceride lipid species in the liraglutide-treated group and we were able to demonstrate that this was primarily a downregulation of large, poly-unsaturated triglycerides. In support of this, when analyzing the lipid species as lipid families, we observed significant reductions in the ceramide, hexocyl-ceramide, phosphatidylcholine, phosphatidylethanolamine and triglyceride 


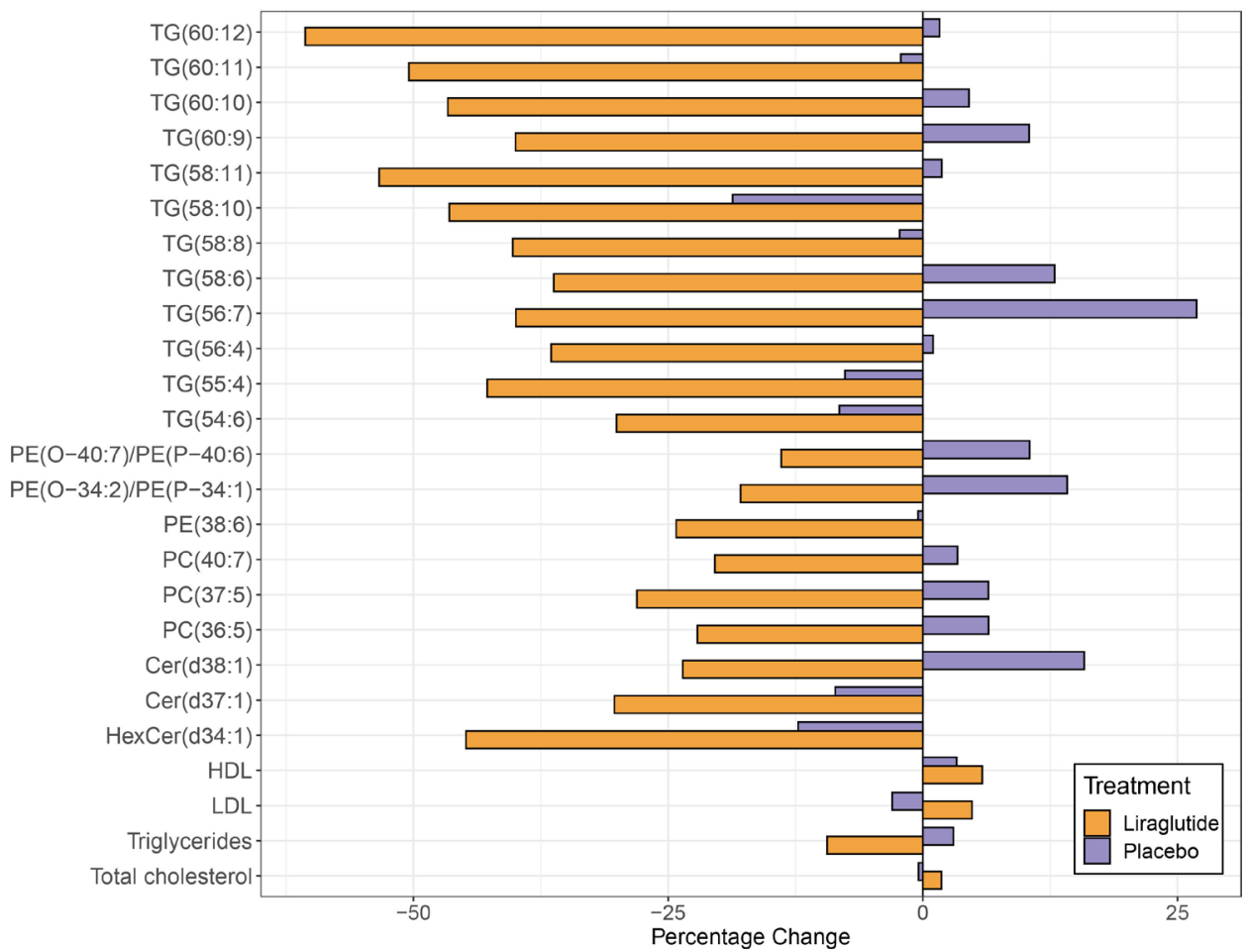

Figure 2 Percentage change after 26 weeks in average amount of individual lipids and in the traditional clinical lipid measurements following liraglutide and placebo treatment. Included are the 21 individual lipids significantly reduced by liraglutide compared with placebo and the traditional clinical lipid measurements, including HDL-cholesterol, LDL-cholesterol, triglycerides and total cholesterol. The traditional clinical lipid measurements were not significantly reduced by liraglutide compared with placebo treatment. Cer, ceramides; HDL, high-density lipoprotein; HexCer, hexocyl-ceramides; LDL, lowdensity lipoprotein; PC, phosphatidylcholines; PE, phosphatidylethanolamines; TG, triglycerides.

lipid family in the liraglutide-treated group compared with placebo. Moreover, mediation analyses indicated that the lipid-regulating effect of liraglutide was likely not mediated by change in BMI or $\mathrm{HbA}_{1 c}$.

Ceramides, phospholipids and triglycerides are lipids identified as biomarkers-and potential causesof cardiovascular disease. Given the robustness and magnitude of the reduction in these pro-atherogenic biomarkers observed, we consider that our findings are interesting, and that liraglutides effect on lipids should be explored further and could improve our understanding of the mechanisms explaining the cardiovascular protection observed with human GLP-1 receptor agonists.

We investigated 260 lipids of which 21 was significantly reduced in the group treated with liraglutide compared with placebo. In the discussion, we focus only on the lipids that changed.

\section{Liraglutide, ceramides and atherosclerosis: a pathway to reduced risk of cardiovascular disease?}

As a novel finding, we demonstrated that ceramide levels were reduced in the group treated with liraglutide. Ceramides are a class of bioactive lipids implicated in cardiovascular disease and with interesting similarities to LDL-cholesterol. Ceramides are present in the serum in much lower concentrations than cholesterol (approximately 1/1000) and can be measured with sensitive techniques such as mass spectrometry. Like cholesterol, ceramides (the backbone of all sphingolipids) accumulate in atherosclerotic lesions. ${ }^{18}$ Experimental data have revealed how ceramides are formed at the surface of atherogenic lipoproteins via sphingomyelinase activity. ${ }^{1920}$ An increased ceramide content promotes lipoprotein aggregation, which in turn promotes the subendothelial retention or trapping of lipoproteins within the vessel wall, a key event in early atherogenesis. ${ }^{19}{ }^{20}$ Ceramides are involved in the transcytosis of lipoproteins across the endothelium ${ }^{21}$ and are further perceived as an important second messenger in several aspects of the inflammatory process. ${ }^{18}$ Several studies have demonstrated that higher circulating levels of ceramides are risk factors for future cardiovascular events in apparently healthy individuals, in individuals with known coronary artery disease as well as in persons with diabetes. ${ }^{22-28}$ Ceramides were recently reported as part of the Cardiovascular Event Risk Test (CERT) and the derivative CERT2 risk-score developed and validated in large cohorts by Hilvo $e t$ al as a simple cardiovascular risk estimation score based on ceramides and phospholipids. ${ }^{29} 30$

In $\mathrm{ApoE}^{-/-}$mice and rabbits fed a high fat and cholesterol diet, inhibiting the biosynthesis of ceramides ameliorates atherosclerosis and arterial stiffness. ${ }^{31}{ }^{32}$ Evidence that therapeutic interventions can reduce ceramides in humans is sparse. The PREDIMED trial recently revealed a strong association between ceramides, and risk 


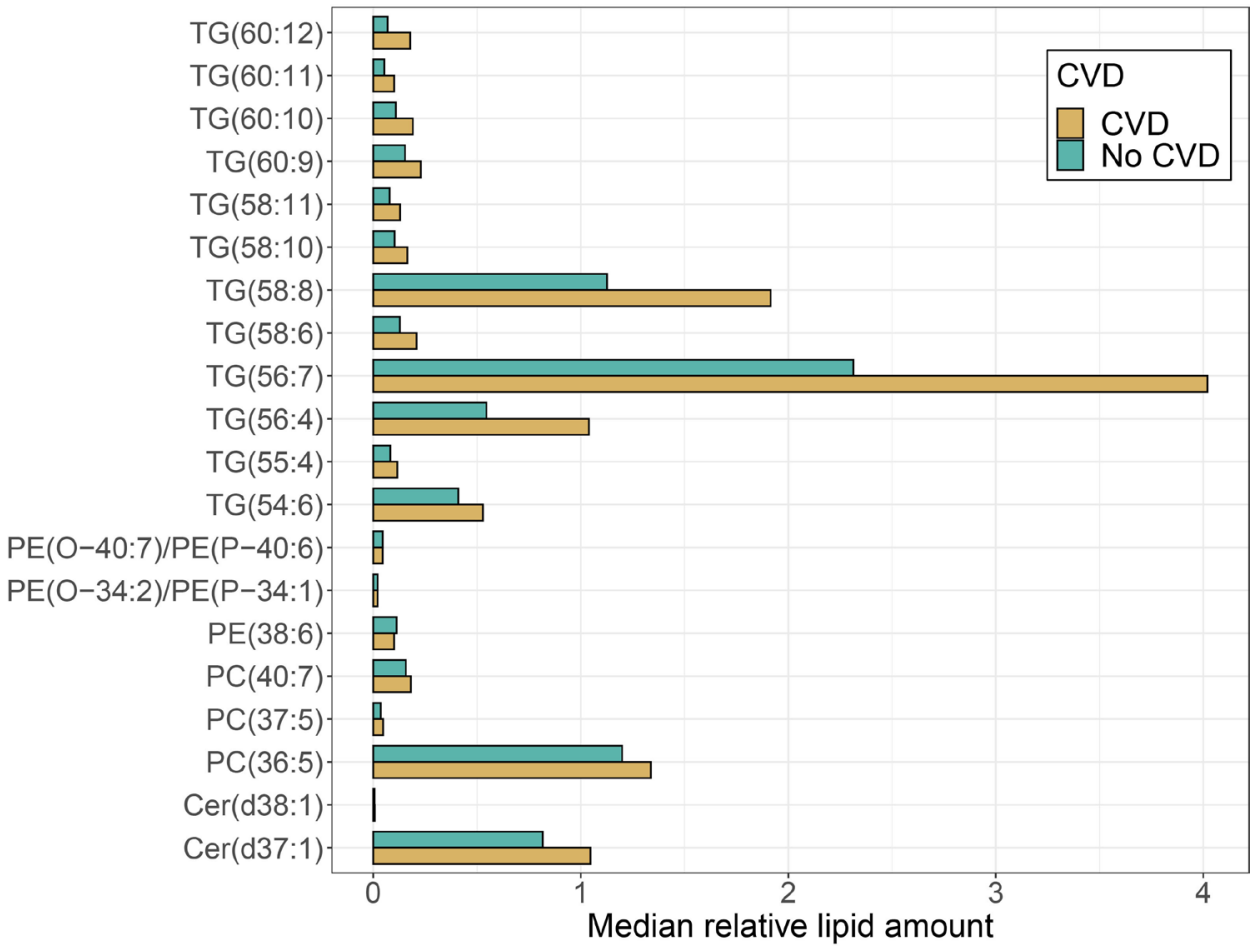

Figure 3 Baseline level of individual lipids for participants with versus without a history of cardiovascular disease. Shown are selected lipids that were significantly reduced by liraglutide compared with placebo treatment for participants with ( $\mathrm{n}=23$ ) vs without a history of cardiovascular disease (CVD). Levels are $\log _{10}$ transformed. Levels were generally higher for participants with a history of cardiovascular disease, although none of the differences were significant. A history of cardiovascular disease was defined as a history of acute myocardial infarction, percutaneous coronary intervention, coronary artery bypass graft, stroke, peripheral arterial thrombosis, claudication and/or nitroglycerin requiring angina pectoris. Cer, ceramides; PC, phosphatidylcholines; PE, phosphatidylethanolamines; TG, triglycerides.

of cardiovascular disease that was mitigated by a Mediterranean dietary intervention. ${ }^{33}$

\section{Liraglutide and phospholipids}

We report significant reductions for the liraglutidetreated group compared with placebo in single-lipid phospholipids from the two lipid families phosphatidylethanolamines and phosphatidylcholines. In comparisons with ceramides, the body of literature that links phospholipids to cardiovascular disease is limited, but emerging. A study in a case-cohort $(\mathrm{n}=3779)$ subset from the Action in Diabetes and Vascular Disease: Preterax and Diamicron-MR trial demonstrated that higher levels of phospholipids among other lipids were associated with risk of cardiovascular morbidity and mortality ${ }^{28}$ and as already mentioned, phospholipids were part of the recently published CERT2 cardiovascular risk estimation score. $^{29}$

\section{Liraglutide and large triglycerides}

Others have demonstrated, before us, that liraglutide lowers the triglyceride level measured as a traditional clinical lipid. ${ }^{54}{ }^{35}$ We identified 88 distinct triglyceride lipid species, which enabled us to add new information that especially large, poly-unsaturated triglycerides were reduced in the liraglutide-treated group. The precise mechanism behind liraglutide's effect on triglycerides has yet to be determined. One hypothesis that has been put forward is that GLP-1 receptor signaling mediates a decreased secretion of apoB48-containing chylomicron particles in the intestinal mucosa, which subsequently reduces the intestinal absorption of triglycerides. ${ }^{3436}$

The overall size of triglyceride reduction with liraglutide has been reported as modest when triglycerides were measured as a traditional clinical lipid. ${ }^{5} 35$ Our data indicate that this modest reduction could cover more pronounced reductions in large, poly-unsaturated triglycerides together with less pronounced effects on other triglyceride lipid species. Our findings were independent of changes in BMI and thus weight changes cannot explain this finding.

It remains controversial that triglyceride reduction could translate into a substantial reduced cardiovascular risk. A long-standing association exists between triglycerides and cardiovascular disease in observational studies, but, whether the triglyceride level is a risk marker for development of cardiovascular disease or more directly promote cardiovascular disease has been discussed for decades. ${ }^{38}$ Mendelian randomization studies support a casual role of triglycerides for risk of cardiovascular disease. ${ }^{39}$ The many randomized clinical trials using medications that lower triglycerides, such as fibrates, niacin and omega-3 fatty acids, have failed to show conclusive evidence of further cardiovascular risk reduction after LDL-cholesterol levels were 'optimally 
controlled'. ${ }^{40}$ However, a positive outcome has recently been reported in the Reduction of Cardiovascular Events with Icosapent Ethyl-Intervention Trial (REDUCE-IT) ${ }^{41}$ In REDUCE-IT, the omega-3 fatty acid product icosapent ethyl reduced the cardiovascular risk regardless of the triglyceride level attained, contributing to the ongoing debate whether triglyceride lowering per se reduces the cardiovascular risk and pointing out to the importance of determining single lipid biochemistry.

\section{Strengths and limitations}

Our study is hypothesis generating. Only similar analysis of a cardiovascular outcome trial could provide evidence that liraglutide prevents cardiovascular events by decreasing ceramides and phospholipids. By using lipidomics we were able to investigate liraglutide's possible effect on a broad panel of lipid families and individual lipids. The strengths of our study include the doubleblinded, randomized, placebo-controlled design and the robust technology applied to measure a comprehensive panel of lipids. Participants were instructed to fast for 4 hours. We can speculate that 12-14hours overnight complete dietary restriction would give lower overall levels of the lipids measured, including triglycerides, but as we are measuring changes from baseline within individuals, we do not think this impacted our findings. Despite the randomized design, we observed a higher baseline triglyceride level in the liraglutide-treated group compared with placebo. As we are measuring changes from baseline within individuals, we do not think this impacts our findings for the single-lipid triglycerides, but we cannot rule out that it is easier to clear more triglycerides with higher levels. We confirmed our findings in a sensitivity analysis, adjusted for the clinical measurement of total triglycerides at baseline. Lipidomics is a tool to study fingerprints with hundreds of lipids but currently it is platform-dependent and does not detect all the lipids in the lipidome. Other relevant variables (ie, glycemic control, body weight) were improved in the liraglutide-treated group, and therefore it is not possible to assume a direct relationship between the observed changes in the single lipids and liraglutide therapy. In order to evaluate if the effect on the lipidome is a direct effect of liraglutide, or secondary to weight loss or improved glycemic control following liraglutide treatment, an active comparator should have been included (ie, insulin as a comparator for liraglutides effect on glycemic control or diet-induced weight loss as a comparator for liraglutides effect on body weight).

\section{Conclusion}

Ceramides, phospholipids and triglycerides were downregulated in the group treated with liraglutide compared with placebo. Lipids which all are linked to risk of cardiovascular disease. This lipid-regulating effect of liraglutide should be examined further and may contribute to the cardiovascular benefits observed in outcome studies.
Acknowledgements The authors are grateful to all the participants and acknowledge the work of study nurse L. Jelstrup and laboratory technicians D. Riis, T. R. Juhl and J. A. Hermann (Steno Diabetes Center Copenhagen, Gentofte, Denmark).

Contributors EHZ, AW, RSR, BJvS, TS, TWH, AK, CL-Q and PR contributed to study design and data interpretation. EHZ recruited participants to the study. EHZ, VRC and TWH contributed to running of the study and data collection. AW performed the lipidomics sample analysis. AW and TS processed and analyzed the lipidomics data. AW, TS and CL-Q interpreted the lipidomics data. EHZ performed statistical analysis of the clinical data. EHZ drafted the manuscript and the final version was critically reviewed and approved by all authors. Guarantor statement: EHZ is the guarantor of the work, and as such, had full access to all the data in the study and takes responsibility for the integrity of the data and the accuracy of the data analysis.

Funding The study was funded by Novo Nordisk and Skibsreder Per Henriksen, R. og hustrus fund. Steno Diabetes Center Copenhagen and Department of Clinical Physiology, Nuclear Medicine \& PET, Rigshospitalet have provided internal funding.

Competing interests AW, VRC, TS and CL-Q declares no competing interests. AK has received consultancy fees from Novo Nordisk. RSR, BJvS, TWH and PR have shares in Novo Nordisk and BJvS and EHZ is now an employee of Novo Nordisk, but work related to this article was conducted while EHZ was employed by Steno Diabetes Center Copenhagen. PR has received the following: consultancy and/ or speaking fees (to Steno Diabetes Center Copenhagen) from AbbVie, Astellas, AstraZeneca, Bayer, Boehringer Ingelheim, Bristol-Myers Squibb, Eli Lilly, MSD, Novo Nordisk and Sanofi Aventis; research grants to institution from AbbVie, AstraZeneca and Novo Nordisk.

Patient consent for publication Not required.

Ethics approval The study was approved by the local ethics committee (H-16044546) and the Danish Medicines Agency (2016110109) and was performed in compliance with the principles of the Declaration of Helsinki.

Provenance and peer review Not commissioned; externally peer reviewed.

Data availability statement Data are available upon reasonable request. The datasets analyzed during the current study are not publicly available due to the risk of patient re-identification. De-identified participant data or anonymised clinical study reports can be obtained from the first author upon reasonable request. Necessary data protection agency and ethical committee approvals must be provided in compliance with relevant legislation.

Supplemental material This content has been supplied by the author(s). It has not been vetted by BMJ Publishing Group Limited (BMJ) and may not have been peer-reviewed. Any opinions or recommendations discussed are solely those of the author(s) and are not endorsed by BMJ. BMJ disclaims all liability and responsibility arising from any reliance placed on the content. Where the content includes any translated material, BMJ does not warrant the accuracy and reliability of the translations (including but not limited to local regulations, clinical guidelines, terminology, drug names and drug dosages), and is not responsible for any error and/or omissions arising from translation and adaptation or otherwise.

Open access This is an open access article distributed in accordance with the Creative Commons Attribution Non Commercial (CC BY-NC 4.0) license, which permits others to distribute, remix, adapt, build upon this work non-commercially, and license their derivative works on different terms, provided the original work is properly cited, appropriate credit is given, any changes made indicated, and the use is non-commercial. See: http://creativecommons.org/licenses/by-nc/4.0/.

\section{ORCID iDs}

Emilie H Zobel http://orcid.org/0000-0003-3318-7070

Asger Wretlind http://orcid.org/0000-0001-6083-5227

Viktor Rotbain Curovic http://orcid.org/0000-0002-4270-6701

\section{REFERENCES}

1 Marso SP, Bain SC, Consoli A, et al. Semaglutide and cardiovascular outcomes in patients with type 2 diabetes. N Engl J Med 2016;375:1834-44.

2 Marso SP, Daniels GH, Brown-Frandsen K. Liraglutide and cardiovascular outcomes in type 2 diabetes. N Engl J Med 2016.

3 Hernandez AF, Green JB, Janmohamed S, et al. Albiglutide and cardiovascular outcomes in patients with type 2 diabetes and cardiovascular disease (harmony outcomes): a double-blind, randomised placebo-controlled trial. Lancet 2018;392:1519-29. 
4 Gerstein HC, Colhoun HM, Dagenais GR, et al. Dulaglutide and cardiovascular outcomes in type 2 diabetes (REWIND): a doubleblind, randomised placebo-controlled trial. Lancet 2019;394:121-30.

5 Nauck MA, Meier JJ, Cavender MA, et al. Cardiovascular actions and clinical outcomes with glucagon-like peptide-1 receptor agonists and dipeptidyl peptidase- 4 inhibitors. Circulation 2017; 136:849-70.

6 Buse JB, Bain SC, Mann JFE, et al. Cardiovascular risk reduction with liraglutide: an exploratory mediation analysis of the LEADER trial. Diabetes Care 2020;43:1546-52.

7 Whiley L, Godzien J, Ruperez FJ, et al. In-vial dual extraction for direct LC-MS analysis of plasma for comprehensive and highly reproducible metabolic fingerprinting. Anal Chem 2012;84:5992-9.

8 Ripa RS, Zobel EH, von Scholten BJ, et al. Effect of liraglutide on arterial inflammation assessed as $\left[^{18} \mathrm{~F}\right] \mathrm{FDG}$ uptake in patients with type 2 diabetes: a randomized, double-blind, placebo-controlled trial. Circ Cardiovasc Imaging 2021;14:e012174.

9 Folch J, Lees M, Sloane Stanley GH. A simple method for the isolation and purification of total lipides from animal tissues. $J$ Biol Chem 1957;226:497-509.

10 Pluskal T, Castillo S, Villar-Briones A, et al. MZmine 2: modular framework for processing, visualizing, and analyzing mass spectrometry-based molecular profile data. BMC Bioinformatics 2010;11:395

11 Tofte N, Suvitaival T, Ahonen L, et al. Lipidomic analysis reveals sphingomyelin and phosphatidylcholine species associated with renal impairment and all-cause mortality in type 1 diabetes. Sci Rep 2019;9:16398

12 Ritchie ME, Phipson B, Wu D, et al. limma powers differential expression analyses for RNA-sequencing and microarray studies. Nucleic Acids Res 2015;43:e47.

13 Bates D, Mächler M, Bolker B. Fitting linear mixed-effects models using Ime4 2015;2015:48.

14 Suvitaival T, Legido-Quigley C. Understanding the lipidome at the systems level with lipidomeR. bioRxiv 2020.

15 Wickham H. ggplot2: elegant graphics for data analysis. New York: Springer-Verlag, 2016.

16 Torchiano M. effsize: efficient effect size computation 2020.

17 Tingley D, Yamamoto T, Hirose K, et al. Mediation: R package for causal mediation analysis 2014;2014:38.

18 Bismuth J, Lin P, Yao Q, et al. Ceramide: a common pathway for atherosclerosis? Atherosclerosis 2008:196:497-504

19 Devlin CM, Leventhal AR, Kuriakose G, et al. Acid sphingomyelinase promotes lipoprotein retention within early atheromata and accelerates lesion progression. Arterioscler Thromb Vasc Biol 2008;28:1723-30.

20 Schissel SL, Tweedie-Hardman J, Rapp JH, et al. Rabbit aorta and human atherosclerotic lesions hydrolyze the sphingomyelin of retained low-density lipoprotein. proposed role for arterial-wall sphingomyelinase in subendothelial retention and aggregation of atherogenic lipoproteins. J Clin Invest 1996;98:1455-64.

21 Li W, Yang X, Xing S, et al. Endogenous ceramide contributes to the transcytosis of oxLDL across endothelial cells and promotes its subendothelial retention in vascular wall. Oxid Med Cell Longev 2014;2014:823071

22 Laaksonen R, Ekroos K, Sysi-Aho M, et al. Plasma ceramides predict cardiovascular death in patients with stable coronary artery disease and acute coronary syndromes beyond LDL-cholesterol. Eur Heart J 2016;37:1967-76.

23 Cheng JM, Suoniemi M, Kardys I, et al. Plasma concentrations of molecular lipid species in relation to coronary plaque characteristics and cardiovascular outcome: results of the ATHEROREMO-IVUS study. Atherosclerosis 2015;243:560-6.
24 Yu J, Pan W, Shi R, et al. Ceramide is upregulated and associated with mortality in patients with chronic heart failure. Can J Cardiol 2015;31:357-63.

25 Havulinna AS, Sysi-Aho M, Hilvo M, et al. Circulating ceramides predict cardiovascular outcomes in the population-based FINRISK 2002 cohort. Arterioscler Thromb Vasc Biol 2016;36:2424-30.

26 Tarasov K, Ekroos K, Suoniemi M, et al. Molecular lipids identify cardiovascular risk and are efficiently lowered by simvastatin and PCSK9 deficiency. J Clin Endocrinol Metab 2014:99:E45-52.

27 Mundra PA, Barlow CK, Nestel PJ, et al. Large-scale plasma lipidomic profiling identifies lipids that predict cardiovascular events in secondary prevention. JCI Insight 2018;3 doi:10.1172/jci. insight. 121326

28 Alshehry ZH, Mundra PA, Barlow CK, et al. Plasma lipidomic profiles improve on traditional risk factors for the prediction of cardiovascular events in type 2 diabetes mellitus. Circulation 2016;134:1637-50.

29 Hilvo M, Meikle PJ, Pedersen ER, et al. Development and validation of a ceramide- and phospholipid-based cardiovascular risk estimation score for coronary artery disease patients. Eur Heart $J$ 2020;41:371-80.

30 Hilvo M, Wallentin L, Ghukasyan Lakic T, et al. Prediction of residual risk by Ceramide-Phospholipid score in patients with stable coronary heart disease on optimal medical therapy. J Am Heart Assoc 2020;9:e015258.

31 Chaurasia B, Summers SA. Ceramides - lipotoxic inducers of metabolic disorders. Trends Endocrinol Metab 2015;26:538-50.

32 Chatterjee S, Bedja D, Mishra S, et al. Inhibition of glycosphingolipid synthesis ameliorates atherosclerosis and arterial stiffness in apolipoprotein E-/- mice and rabbits fed a high-fat and -cholesterol diet. Circulation 2014;129:2403-13.

33 Wang DD, Toledo E, Hruby A, et al. Plasma ceramides, Mediterranean diet, and incident cardiovascular disease in the PREDIMED trial (Prevencion con Dieta Mediterranea). Circulation 2017; 135:2028-40.

34 Hermansen K, Bækdal TA, Düring M, et al. Liraglutide suppresses postprandial triglyceride and apolipoprotein B48 elevations after a fat-rich meal in patients with type 2 diabetes: a randomized, doubleblind, placebo-controlled, cross-over trial. Diabetes Obes Metab 2013;15:1040-8.

35 Buse JB, Nauck M, Forst T, et al. Exenatide once weekly versus liraglutide once daily in patients with type 2 diabetes (DURATION-6): a randomised, open-label study. Lancet 2013;381:117-24.

36 Hsieh J, Longuet C, Baker CL, et al. The glucagon-like peptide 1 receptor is essential for postprandial lipoprotein synthesis and secretion in hamsters and mice. Diabetologia 2010;53:552-61.

37 Sun F, Wu S, Wang J, et al. Effect of glucagon-like peptide-1 receptor agonists on lipid profiles among type 2 diabetes: a systematic review and network meta-analysis. Clin Ther 2015;37:e228:225-41.

38 Miller M, Stone NJ, Ballantyne C, et al. Triglycerides and cardiovascular disease: a scientific statement from the American heart association. Circulation 2011:123:2292-333.

39 Holmes MV, Asselbergs FW, Palmer TM, et al. Mendelian randomization of blood lipids for coronary heart disease. Eur Heart $J$ 2015;36:539-50.

40 Ganda OP, Bhatt DL, Mason RP, et al. Unmet need for adjunctive dyslipidemia therapy in hypertriglyceridemia management. J Am Coll Cardiol 2018;72:330-43.

41 Bhatt DL, Steg PG, Miller M, et al. Cardiovascular risk reduction with Icosapent ethyl for hypertriglyceridemia. N Engl J Med 2019;380:11-22. 